\title{
Recurrence Spectroscopy of a Time-Dependent System: A Rydberg Atom in an Oscillating Field
}

\author{
Neal Spellmeyer \\ Daniel Kleppner \\ M. R. Haggerty \\ William \& Mary \\ V. Kondratovich \\ William \& Mary \\ John B. Delos \\ William \& Mary, jbdelos@wm.edu
}

See next page for additional authors

Follow this and additional works at: https://scholarworks.wm.edu/aspubs

Part of the Physics Commons

\section{Recommended Citation}

Spellmeyer, Neal; Kleppner, Daniel; Haggerty, M. R.; Kondratovich, V.; Delos, John B.; and Gao, J., Recurrence Spectroscopy of a Time-Dependent System: A Rydberg Atom in an Oscillating Field (1997). Physical Review Letters, 79(9), 1650-1653.

https://doi.org/10.1103/PhysRevLett.79.1650

This Article is brought to you for free and open access by the Arts and Sciences at W\&M ScholarWorks. It has been accepted for inclusion in Arts \& Sciences Articles by an authorized administrator of W\&M ScholarWorks. For more information, please contact scholarworks@wm.edu. 
Authors

Neal Spellmeyer, Daniel Kleppner, M. R. Haggerty, V. Kondratovich, John B. Delos, and J. Gao 


\title{
Recurrence Spectroscopy of a Time-Dependent System: A Rydberg Atom in an Oscillating Field
}

\author{
Neal Spellmeyer and Daniel Kleppner \\ Research Laboratory of Electronics, George R. Harrison Spectroscopy Laboratory, and Department of Physics, \\ Massachusetts Institute of Technology, 77 Massachusetts Avenue, Cambridge, Massachusetts 02139 \\ M. R. Haggerty, V. Kondratovich, and J. B. Delos \\ Physics Department, College of William and Mary, Williamsburg, Virginia 23187 \\ J. Gao \\ Chemistry and Physics Department, Kean College, Union, New Jersey 07083
}

(Received 28 April 1997)

\begin{abstract}
We report the results of an experimental and theoretical investigation of the recurrence spectra of Rydberg atoms in a static plus weak oscillating electric field. Experiments reveal the systematic weakening of orbits in a recurrence spectrum as the oscillating field strength and frequency are changed. We describe a generalization of closed orbit theory to time-dependent systems and show that it provides a qualitative and quantitative description of the phenomena. [S0031-9007(97)03947-1]
\end{abstract}

PACS numbers: 32.60. $+\mathrm{i}, 03.65 . \mathrm{Sq}, 05.45 .+\mathrm{b}$

In the quest for a satisfactory understanding of the connections between quantum and classical descriptions of simple Hamiltonian systems, Rydberg atoms in applied fields provide prototypes for experiment and theory [1]. Recurrence spectroscopy and closed orbit theory have proven to be powerful tools for these studies [2-4]. A recurrence spectrum is the Fourier transform of a photoexcitation spectrum that is taken with the field varying with energy according to a classical scaling rule that keeps the classical motion unchanged at all points in the spectrum [2]. It can be shown that each closed classical orbit of the electron generates a peak in the recurrence spectrum at the action of the orbit. Consequently, the recurrence spectrum provides a quantum picture of classical behavior. Studies of recurrence spectra have led to observations of the creation of new orbits through bifurcations $[5,6]$, the onset of irregular behavior through core scattering [7-10], symmetry breaking in crossed fields [11], and the identification of numerous closed orbits [1].

We have extended this line of inquiry by investigating the recurrence spectrum of a Rydberg atom in a field that is oscillating with a period which is comparable to the period of its classical orbits. The underlying thought is that periodic orbits should be sensitive to periodic perturbations, and that this sensitivity should be revealed by recurrence spectra. Recurrences with periods that are integer multiples of the period of the perturbing field might be expected to be most affected. We report here the first results of such a study. The experimental observations are surprising: Recurrences with periods near integer multiples of the period of the perturbation survive, while those that are out of "resonance" with the field are weakened or eliminated. We have been able to interpret these results by generalizing closed orbit theory to incorporate periodic fields, obtaining both a qualitative and quantitative description of the phenomena.

Our study employs a lithium Rydberg atom in a static electric field - a system we have previously studied [8] but now with the addition of an oscillating field. At the low actions studied in these experiments, the spectrum is regular and can be understood by considering only the closed orbits of hydrogen. Experimentally, introducing an rf field is a straightforward task. (We use "rf" to signify the oscillating field though the actual frequency may be in the microwave regime.)

The Hamiltonian of a Rydberg atom in an electric field $F$ along the $z$ axis and an $\mathrm{rf}$ field with amplitude $F_{1}$ oscillating at angular frequency $\omega$, also polarized along the $z$ axis, is taken to be (in atomic units)

$$
H=\frac{p^{2}}{2}-\frac{1}{r}+F z+F_{1} z \cos (\omega t) .
$$

Recurrence spectroscopy is possible because the classical Hamiltonian can be expressed completely in scaled variables. We define $\tilde{r}=F^{1 / 2} r, \tilde{p}=F^{-1 / 4} p, \tilde{t}=F^{3 / 4} t$, $\tilde{\omega}=F^{-3 / 4} \omega$, and $\tilde{f}=F^{-1} F_{1}$. This yields the scaled Hamiltonian

$$
\tilde{H}=\frac{\tilde{p}^{2}}{2}-\frac{1}{\tilde{r}}+\tilde{z}[1+\tilde{f} \cos (\tilde{\omega} \tilde{t})]=F^{-1 / 2} E(t) .
$$

In the oscillating field, the electron energy $E(t)$ is not constant. We define $\epsilon \equiv F^{-1 / 2} E^{\text {out }}$ to be the scaled energy of the electron as it leaves the atom. The scaled action is $\tilde{S}=F^{1 / 4} S$ [12]. To obtain a recurrence spectrum we simultaneously vary the laser energy, static and rf field amplitudes, and the rf frequency so as to keep the scaled parameters $(\epsilon, \tilde{f}$, and $\tilde{\omega})$ constant as we record the photoabsorption spectrum.

The experimental setup is similar to that described in [8]. Lithium is excited to the $3 s$ state by two-step 
resonant excitation $2 s \rightarrow 2 p \rightarrow 3 s$ and then to an $m=0$ Rydberg state by a tunable laser. The scaled energy and frequency are known to an accuracy of about $0.1 \%$. The rf field amplitude was calibrated by measuring sidebands on various states in a Stark manifold [13], yielding an overall accuracy in $F_{1}$ of $10 \%$, with $1 \%$ accuracy at selected calibration points. In our experiments, we measure actions up to $\tilde{S} \approx 150$.

We chose to study behavior at scaled energy $\epsilon=-0.4$, which we had mapped previously for a static electric field [6]. Because this energy lies above the saddle point threshold for ionization $(\epsilon=-2)$, orbits directed towards the negative $z$ direction are absent. The most important orbit is the parallel "uphill" orbit lying along the positive $z$ axis, and its repetitions. These form a spectrum of recurrences equally spaced in action at an interval $\Delta \tilde{S}=4.337$. The second repetition lies close to a bifurcation point at $\epsilon=-0.397$ [14], which strongly enhances its recurrence strength. In contrast, the first repetition is extremely weak. We mapped recurrence spectra with constant scaled amplitude $\tilde{f}$ and also constant scaled frequency $\tilde{\omega}$.

Figure 1 displays a panoramic map of recurrence spectra at $\tilde{f}=0.005$, with $\tilde{\omega}$ varying from 0.12 to 0.60 . The most conspicuous feature is a series of regions where the recurrences are practically annihilated and a series of regions where they survive. The periods of the surviving orbits are near integer multiples of $2 \pi / \tilde{\omega}$, shown by dark lines in the plot. In addition, the strong second repetition persists at all frequencies measured.

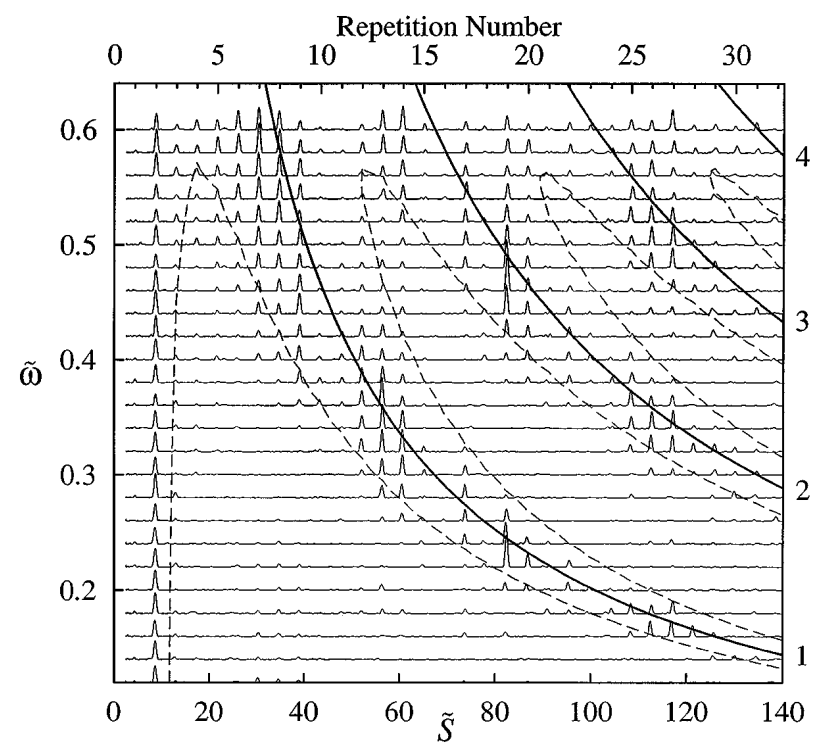

FIG. 1. Experimental recurrence spectra recorded at $\epsilon=$ -0.4 and $\tilde{f}=0.005$, with scaled frequency $\tilde{\omega}$ between 0.12 and 0.60 in steps of 0.02 . The dashed lines are contours generated from Eq. (10) where $a_{n}=0.2$. The solid curved lines mark the action at which a repetition of the parallel orbit has a period that is an integer multiple $n$ of the period of the applied field. The values of $n$ are listed on the right.
Figure 2 displays a map at fixed frequency $\tilde{\omega}=0.32$, with $\tilde{f}$ varying from 0.0 to 0.022 . As $\tilde{f}$ increases, all the peaks lose strength. Some recurrences are very sensitive and fall off rapidly as $\tilde{f}$ increases; others persist to much higher $\tilde{f}$. As the rf field is made yet stronger, many of the peaks revive, some more than once.

The peaks in Figs. 1 and 2 are manifestations of recurrences-classical orbits which go out from the nucleus and return. To interpret the data, we briefly review the ideas of closed orbit theory and then discuss its extension to a time-periodic Hamiltonian. The theory of recurrences begins from the usual quantum theory of photoexcitation: As the laser radiation causes transitions between the initial state and high energy eigenstates of $H$, there is a well-defined rate of absorption. The smoothed or large scale structure of the spectrum can be expressed as an oscillator strength density, which we denote $D f(E, F)$. The oscillator strength density can be separated into two parts:

$$
D f(E, F)=D f_{0}+D f_{1}(E, F) .
$$

$D f_{0}$ is a smooth (practically constant) background that is approximately the same as for the field-free atom at the ionization threshold. $D f_{1}(E, F)$, which varies rapidly with $E$ and $F$, is due to recurrences that arise in the following manner. The laser radiation produces a stream of outgoing waves which, in the semiclassical approximation, follow classical trajectories. As the trajectories travel outward, some are turned back by the combined Coulomb and applied fields and return to the atom. These interfere with the outgoing waves (and with each other), giving rise to oscillatory patterns in the absorption spectrum. Each returning orbit $k$ gives a sinusoidal

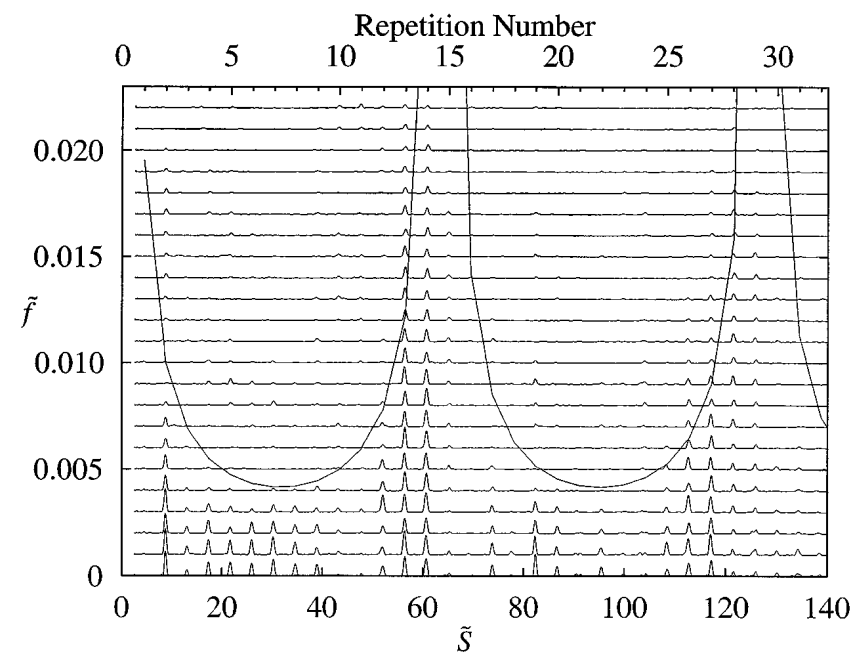

FIG. 2. Experimental recurrence spectra recorded at $\epsilon=$ -0.4 and $\tilde{\omega}=0.32$ with scaled rf field amplitude $\tilde{f}$ between 0.0 and 0.022 . The solid curved lines mark the location of the first zero in the recurrence strength as predicted by the restricted semiclassical theory, Eq. (10). 
contribution to the oscillator strength density,

$$
D f_{1, k}(E, F)=C_{k} \sin \left[S_{k}^{0}(E, F) / \hbar+\gamma_{k}\right] .
$$

The corresponding expression in scaled variables is

$$
D f_{1, k}(\epsilon, w)=C_{k} \sin \left[\tilde{S}_{k}^{0}(\epsilon) w+\gamma_{k}\right] .
$$

$S_{k}^{0}(E, F) \equiv \int \mathbf{p} \cdot d \mathbf{q}$ is the classical action around the closed orbit, $\tilde{S}_{k}^{0}(\epsilon)$ is its scaled counterpart $w=F^{-1 / 4}$, and $\gamma_{k}$ is a phase correction associated with Maslov indices. $C_{k}$ is the recurrence amplitude, which is approximately independent of $E$ and $F$. The absolute square of the Fourier transform of the absorption spectrum with respect to $w$ gives the recurrence spectrum, which reveals peaks at the scaled actions of the closed orbits.

We have generalized closed orbit theory to incorporate a time-dependent Hamiltonian. We summarize the main features here- a full description will be published elsewhere. The explicit time dependence of the Hamiltonians in Eqs. (1) and (2) means that many aspects of recurrence theory need to be reexamined. It can be shown [15] that at each time $t$, it is possible to define an instantaneous oscillator strength density $D f\left(E, F ; F_{1}, \omega, t\right)$ out of the initial state into a band of excited states. $D f$ can be separated again as in Eq. (3), with $D f_{1}$ arising from closed orbits. The physical picture is much as before. The laser produces a steady stream of outgoing waves, which now propagate in the oscillating electric field. The result is a time-dependent wave function that in the semiclassical description is "supported" by time-dependent classical trajectories. Each returning orbit in the static field represents a continuous family of identical orbits, each starting at a different time. With the oscillating field turned on, the orbits in a family are no longer identical because their properties then depend on the phase of the rf field when they left the atom. We call this splitting of a single orbit into a continuous family of orbits "temporal symmetry breaking," because of its close analogy to cylindrical symmetry breaking $[11,15]$. The component of $D f_{1}$ arising from each family oscillates at the rf frequency, and the measurement averages it over many cycles.

The returning wave can be calculated using a semiclassical approximation in an extended phase space $(\mathbf{p}, \mathbf{q}, E, t)$ where time is regarded as a dynamical variable. The conjugate variable $E$ is not conserved because of the temporal symmetry breaking. The action associated with the returning wave is again the classical action, but now in the extended phase space:

$$
R(t)=\int\left\{\mathbf{p} \cdot d \mathbf{q}-\left[H(t)-E^{\mathrm{out}}\right] d t\right\} .
$$

This generalized action depends on the phase of the field relative to the orbit. For some phases, the orbit is stretched and its action increases, while for others, the orbit is compressed, and its action decreases. At small rf amplitudes, there is a smooth sinusoidal change between these two extremes. Using first order classical perturbation theory, the generalized action is found to be

$$
R_{k}(t) \approx S_{k}^{0}(E, F)+F_{1}\left|Z_{k}(\omega)\right| T_{k} \cos \left(\omega t-\alpha_{k}\right),
$$

where $T_{k}$ is the period of the orbit, $Z_{k}(\omega)$ is the timeaveraged ac electric dipole moment of the unperturbed orbit $\left(1 / T_{k}\right) \int_{0}^{T_{k}} z(\tau) e^{-i \omega \tau} d \tau$, and $\alpha_{k}$ is the phase of the rf field for which the maximal action is attained.

Substituting Eq. (7) into Eq. (5), averaging over a cycle of the field, and recasting it in scaled variables yields

$$
D f_{1, k}=C_{k} J_{0}\left(\tilde{f}\left|\tilde{Z}_{k}(\tilde{\omega})\right| \tilde{T}_{k} w\right) \sin \left[\tilde{S}_{k}^{0}(\epsilon) w+\gamma_{k}\right] .
$$

This result provides a general description of the absorption spectrum of a Rydberg atom in a weak oscillating electric field. Comparing Eq. (8) with Eq. (5), we see that the perturbation weakens the recurrence amplitude by a factor given by a Bessel function. The argument of the Bessel function is the perturbation to the semiclassical phase of the extreme orbits in the family - proportional to the product of the perturbing field and the ac dipole moment of the unperturbed orbit.

In principle one must evaluate $\tilde{Z}_{k}(\tilde{\omega})$ separately for each unperturbed closed orbit. However, in the regime observed here, the $z$ motion of every orbit is similar to that of the parallel orbit or one of its repetitions. We approximate $\tilde{Z}_{k}(\tilde{\omega})$ by that of the corresponding repetition of the parallel orbit in what we call the restricted semiclassical theory. Additionally, the ac dipole moment for the $n$th repetition of the parallel orbit is related to that for the 1st repetition:

$$
\left|\tilde{Z}_{n}(\tilde{\omega})\right|=\left|\tilde{Z}_{1}(\tilde{\omega})\right|\left|\frac{\sin \left(n \tilde{\omega} \tilde{T}_{1} / 2\right)}{\sin \left(\tilde{\omega} \tilde{T}_{1} / 2\right)}\right| .
$$

Using these approximations in (8), we find that every recurrence peak is weakened by a factor $a_{n}$ given by

$$
a_{n}=J_{0}^{2}\left(\tilde{f} \tilde{Z}_{1}(\tilde{\omega})\left|\frac{\sin \left(n \tilde{\omega} \tilde{T}_{1} / 2\right)}{\sin \left(\tilde{\omega} \tilde{T}_{1} / 2\right)}\right| \tilde{T}_{1} w\right) \equiv J_{0}^{2}\left(c_{n} \tilde{f}\right) .
$$

(In our experiment the range of $w$ is small so it can be taken to be a constant.) We shall refer to the quantity $c_{n}$ defined in Eq. (10) as the falloff coefficient.

We now can examine Figs. 1 and 2 in detail. The dashed lines in Fig. 1 are contours generated from Eq. (10) that show where the recurrence strength should be reduced by $80 \%\left(a_{n}=0.2\right)$. The solid line in Fig. 2 shows the location of the first zero of the Bessel function $c_{n} \tilde{f} \approx 2.4$. This simple calculation predicts the large scale features in the data, and even some details. To further test the experiment and semiclassical theory, we have compared our results with approximate quantum mechanical Floquet calculations of the recurrence spectrum. Details of these calculations will be published elsewhere [16]. Figure 3 compares the experimental recurrence strength of the second repetition in Fig. 2 with the prediction of Eq. (10). The experimental results and 


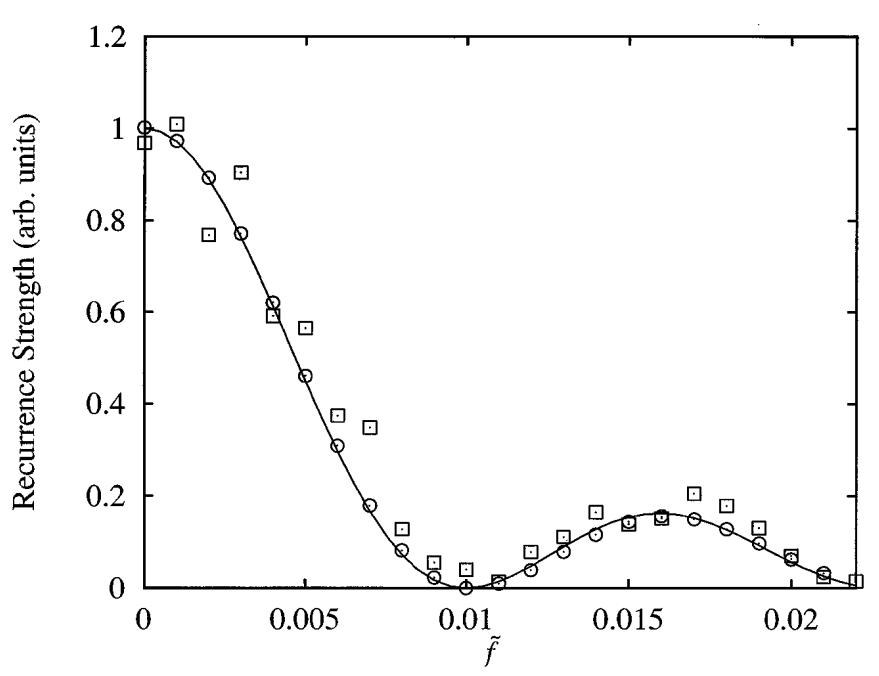

FIG. 3. Recurrence strength of the second repetition at $\epsilon=$ -0.4 and $\tilde{\omega}=0.32$ vs scaled rf field amplitude, $\tilde{f}$. Squares are experimental recurrence strengths, circles are from the Floquet computation. The solid line is the prediction of Eq. (10), scaled to agree near $\tilde{f}=0$.

the two theoretical calculations are in generally good agreement.

The restricted semiclassical theory neglects the differences between the parallel and nonparallel orbits. These differences produce, for example, the small discrepancies between the experiment and restricted semiclassical theory seen in Fig. 2. We have included the exact properties of the nonparallel orbits in what we call the unrestricted semiclassical theory. A convenient way to summarize the results is to fit them to the form of Eq. (10) and express the result in terms of the falloff coefficients $c_{n}$. Values of $c_{n}$ from the experiment, the Floquet computation, and the unrestricted semiclassical model are compared with Eq. (10) in Fig. 4. The restricted semiclassical model can be seen to provide a good approximate picture, which is made even better by the unrestricted calculation.

Our recurrence spectra show the systematic elimination of recurrences. We have confirmed these effects in quantum mechanical Floquet calculations. We have shown how these results can be explained to be a consequence of the destructive averaging of orbits returning to the nucleus at different times. These results provide a firm foundation for the investigation and interpretation of recurrence spectra in time-periodic potentials.

The work at MIT is supported by NSF Grant No. PHY9221489 and ONR Grant No. N00014-96-1-0484, and the work at W\&M by NSF Grant No. PHY-9630372 and ONR Grant No. N00014-94-1-0930. N. S. acknowledges support by an AASERT fellowship from ONR. J.G. acknowledges support from the donors of the Petroleum Research Fund administrated by the American Chemical Society and from an award from Research Corporation.

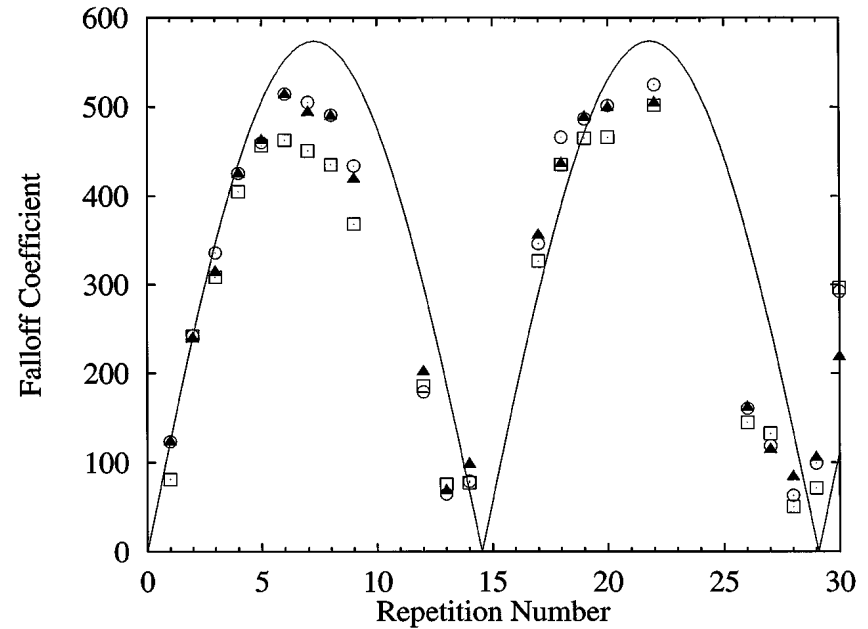

FIG. 4. Falloff coefficient $c_{n}$ [defined in Eq. (10)] for the first 30 repetitions of the parallel orbit. Squares are experiment, circles are Floquet computation, and solid triangles are the unrestricted semiclassical theory. The solid line is the prediction of the restricted semiclassical theory.

The authors thank the Alexander-von-Humboldt-Stiftung for partial support of this work under the Max-PlanckForschungspreis program.

[1] A collection of recent work is given in the Special Issue on Atoms in External Fields, edited by J.P. Connerade [J. Phys. B 27, 2659 (1994)].

[2] A. Holle, J. Main, G. Wiebusch, H. Rottke, and K. H. Welge, Phys. Rev. Lett. 61, 161 (1988).

[3] M. L. Du and J. B. Delos, Phys. Rev. A 38, 1896 (1988).

[4] E. B. Bogomol'nyi, Zh. Eksp. Teor. Fiz. 96, 487 (1989) [Sov. Phys. JETP 69, 275 (1989)].

[5] J. Main, G. Wiebusch, K. Welge, J. Shaw, and J. B. Delos, Phys. Rev. A 49, 847 (1994).

[6] M. Courtney, H. Jiao, N. Spellmeyer, D. Kleppner, J. Gao, and J. B. Delos, Phys. Rev. Lett. 74, 1538 (1995).

[7] M. Courtney, H. Jiao, N. Spellmeyer, and D. Kleppner, Phys. Rev. Lett. 73, 1340 (1994).

[8] M. Courtney, N. Spellmeyer, H. Jiao, and D. Kleppner, Phys. Rev. A 51, 3604 (1995).

[9] P. A. Dando, T. S. Monteiro, D. Delande, and K. T. Taylor, Phys. Rev. Lett. 74, 1099 (1995).

[10] B. Hüpper, J. Main, and G. Wunner, Phys. Rev. Lett. 74, 2650 (1995).

[11] C. Neumann, R. Ubert, S. Freund, E. Flöthmann, B. Sheehy, K. H. Welge, M. R. Haggerty, and J. B. Delos, Phys. Rev. Lett. 78, 4705 (1997).

[12] We omit a factor of $2 \pi$ often used in this subfield.

[13] H. B. van Linden van den Heuvell, R. Kachru, N. H. Tran, and T.F. Gallagher, Phys. Rev. Lett. 53, 1901 (1984).

[14] J. Gao and J. B. Delos, Phys. Rev. A 49, 869 (1994).

[15] M. Haggerty et al. (to be published).

[16] V. Kondratovich et al. (to be published). 